\title{
PREVALÊNCIA DE DESVIO FONOLÓGICO EM CRIANÇAS DE 4 A 6 ANOS DE ESCOLAS MUNICIPAIS DE EDUCAÇÃO INFANTIL DE CANOAS RS
}

\author{
Prevalence of phonological deviations in children - 4 to 6 year old - \\ from a kindergarten school in Canoas - RS
}

\author{
Camila dos Santos Indrusiak ("), Sheila Petry Rockenbach (2)
}

\begin{abstract}
RESUMO
Objetivo: verificar a prevalência de desvio fonológico e processos fonológicos quanto ao sexo e a idade. Método: análise estatística e descritiva da Avaliação Fonológica Infantil de Yavas, Hernadorema e Lamprecht (2002), realizada com 60 pré-escolares de 4 a 6 anos de idade, de Escolas Municipais de Educação Infantil de Canoas-RS. A amostra foi composta por crianças cujos pais não relataram no questionário alterações auditivas, neurológicas, síndromes ou que estivessem realizando ou já tivessem realizado tratamento fonoaudiológico e/ou apresentassem alterações de origem fonética. Resultados: a prevalência de desvio fonológico foi de $55 \%$, sendo maior para o sexo masculino. A maior prevalência de desvio fonológico foi notada com crianças de 5 anos de idade. Os processos fonológicos mais encontrados foram de redução de encontro consonantal (46, 7\%), apagamento de líquida final (40\%) e substituição de líquida (30\%). A relação da prevalência de processo fonológico com o sexo se mostrou de forma semelhante para ambos os sexos, com exceção do apagamento de sílaba átona que foi significante para o sexo masculino. Conclusão: a alta prevalência de desvio fonológico aponta a necessidade de inserção de programas públicos de promoção e prevenção à saúde da comunicação humana na atenção primária.
\end{abstract}

DESCRITORES: Prevalência; Fonoaudiologia; Linguagem

\section{INTRODUÇÃO}

No Brasil, ainda são poucos os estudos fonoaudiológicos voltados para a área de epidemiologia. $\mathrm{A}$ Fonoaudiologia necessita de estudos que tenham como finalidade verificar a prevalência de distúrbios da comunicação humana, para que, embasados em dados científicos, possa promover ações de Saúde Pública e assim delimitar seus campos de atuação ${ }^{1}$. É referido na literatura que, dentre as alterações de fala, o desvio fonológico é caracterizado

(1) Fonoaudióloga pelo Curso de Fonoaudiologia da Universidade Luterana do Brasil-ULBRA,Canoas, RS, Brasil.

(2) Fonoaudióloga; Professora adjunta do Curso de Fonoaudiologia da Universidade Luterana do Brasil - ULBRA, Canoas, Rio Grande do Sul, Brasil; Mestre em Ciências Médicas pela Universidade Federal do Rio Grande Do Sul - UFRGS.

Conflito de interesses: inexistente como um dos mais freqüentes na população infantil. Pesquisa realizada em uma clínica-escola constatou que, entre as alterações de fala, $72,5 \%$ eram desvios fonológicos ${ }^{2}$.

Um estudo realizado no Rio Grande do Sul, em Escolas Municipais da cidade de Canoas, relatou que a prevalência de desvios fonético-fonológicos na população estudada foi de $24,6 \%^{3}$. Pesquisa realizada em oito cidades do Vale do Paraíba constatou a prevalência de crianças com alteração fonológica foi de $8,27 \%{ }^{4}$, e os processos fonológicos mais observados foram simplificação de encontro consonantal $(60,67 \%)$ e simplificação de líquidas $(47,19 \%)^{3,4}$.

Desvio fonológico é uma alteração na organização do sistema fonológico que interfere no desenvolvimento normal da fala, podendo não resultar em alterações de outros níveis da comunicação, como na escrita, na leitura e, até mesmo, na inserção e 
ascensão social ${ }^{3-5}$. Caracteriza-se o distúrbio ou desvio fonológico quando os processos fonológicos (redução de encontro consonantal, apagamento de sílaba átona, apagamento da fricativa final, apagamento da líquida final, apagamento da líquida, apagamento da líquida intervocálica, metátese, epêntese, dessonorização, anteriorização, substituição de líquida, plosivização, posteriorização, assimilação, sonorização pré vocálica), são usados de forma desviante, bem como o uso inadequado de regras fonológicas da língua. Regras fonológicas expressam os processos pelos quais a língua passa, de forma que as representações subjacentes tornem-se representações fonéticas, enquanto processos fonológicos dizem respeito à simplificações das regras fonológicos que envolvem sequências de sons da fala ${ }^{5,6,8}$. Este transtorno é observado em crianças as quais apresentam alterações na produção da fala, na ausência de determinados fatores etiológicos, tais como: dificuldade geral de aprendizagem, deficiência auditiva, atraso na aquisição e desenvolvimento da linguagem com ou sem comprometimento cognitivo, desordem neuromotora, distúrbios psiquiátricos, dentre outros ${ }^{4,6}$.

A aquisição do sistema fonológico ocorre de maneira gradual até o estabelecimento do mesmo. Está relacionada ao processo de desenvolvimento da linguagem normal e com a comunidade lingüística na qual a criança está inserida. Resultados de estudos acerca do desenvolvimento fonológico referem que este deve estar praticamente completo aos cinco anos, dado este controverso na literatura, na qual encontra-se que esse período pode se estender até os sete anos de idade ${ }^{3-5,7,8}$.

Em crianças com desenvolvimento fonológico normal, os fonemas plosivos e nasais são os primeiros segmentos consonantais a serem adquiridos, estando presente geralmente antes dos dois anos de idade. Os fonemas fricativos, na ordem da aquisição fonológica, seguem-se aos fonemas plosivos e nasais. Na classe dos fonemas fricativos, aqueles de aquisição inicial são /f/ e / $/ \mathrm{v}$, enquanto os de aquisição tardia como /s/, /z/, /sh/, /zh/. Os fonemas sonoros são adquiridos antes dos fonemas coocorrentes surdos. Os fonemas africados são adquiridos um pouco mais tardiamente, entre três anos e seis meses e quatro anos e seis meses. A classe das líquidas é a última a ser adquirida, sendo as líquidas laterais adquiridas antes das líquidas não laterais, uma vez que dentro desta classe o /// é adquirido antes do $/ N$, e o /R/ antes do /r/. Em relação à estabilização da produção do $/ r /$, estudos apontam a idade de sete anos, expandindo o limite de aquisição normal do sistema fonológico ${ }^{8-10}$.

Não se tem um consenso a respeito do número de processos fonológicos possíveis. Pesquisas com crianças normais falantes do português brasileiro apontam treze processos fonológicos como os mais comuns, os quais ocorrem no período entre um ano e seis meses e cinco anos de idade 5,10, 11 .

Existem controvérsias no que diz respeito à idade na qual ocorre a supressão dos processos fonológicos, segundo Vitor e Cardoso-Martins (2007) e estudos mais recentes relatam que o desenvolvimento fonológico do português brasileiro deve estar praticamente completo aos cinco anos ${ }^{4,5}$, podendo também ocorrer próximo ao início do processo de alfabetização, não ultrapassando os sete anos de idade. No decorrer do desenvolvimento normal da aquisição fonológica são observados processos fonológicos de omissão e substituição, entre outros, que devem desaparecer ao longo dos anos pré-escolares.

A relevância deste projeto reside no fato de se tratar de um estudo epidemiológico, com vistas a fornecer subsídios para futuros programas de Saúde Pública, principalmente no que diz respeito à promoção e prevenção de alterações no âmbito da linguagem na primeira infância, pois teve em vista desenvolver um estudo epidemiológico e poderá fornecer subsídios para futuros programas de Saúde Pública em promoção e prevenção de alterações da saúde da comunicação humana, na primeira infância.

O objetivo geral da pesquisa foi verificar a prevalência de desvios fonológicos em pré-escolares de quatro a seis anos de idade, de Escolas Municipais de Educação Infantil, no município de Canoas-RS. Como objetivos específicos, temos: (1) avaliar quais são os processos fonológicos alterados com maior frequência na fala dos pré-escolares e (2) relacionar a prevalência dos desvios fonológicos e processos fonológicos com idade e gênero.

\section{MÉTODO}

Nesta pesquisa foi realizado um estudo prospectivo, descritivo, quantitativo, transversal e de grupo.

A amostra foi selecionada na rede de Escolas Municipais de Educação Infantil de Canoas que é composta por 31 escolas, sendo estas divididas em quatro quadrantes, quadrante 1 Sudoeste, quadrante 2 Sudeste, quadrante 3 Nordeste e quadrante 4 Noroeste.

Foram sorteadas, junto à Secretaria de Educação Infantil, quatro escolas localizadas uma em cada quadrante. Em cada escola foram selecionados em média 25 alunos, por meio de sorteio realizado pela pesquisadora na presença da direção da escola. Foram incluídos todos os alunos de 4 a 6 anos, independente da turma ou turno em que estudam. Os alunos foram divididos por gênero, para que desta 
maneira se obtivesse uma proporção de crianças sorteadas do sexo feminino e masculino.

Foi entregue aos participantes da pesquisa um termo de consentimento livre e esclarecido, informando que não haveria riscos às pessoas a ela submetidas, com a de sigilo dos dados. Os participantes tiveram liberdade para participar ou não e interromperem a participação na pesquisa quando necessitassem. Foram excluídas da amostra as crianças cujos responsáveis referiram no questionário a presença de alterações neurológicas, queixa de falta de audição, quadro sindrômico, qualquer patologia que poderia afetar a linguagem da criança e/ou que tenham realizado ou estivessem realizando terapia fonoaudiológica, e crianças que na triagem de motricidade orofacial apresentaram desvios fonéticos.

Participaram da pesquisa 70 crianças, dentre estas, 60 foram incluídas na amostra, as 10 restantes foram excluídas por apresentarem alterações de origem neurológica, auditiva, síndromes, alterações de fala de origem fonética e que já realizaram e/ou estivessem realizando tratamento fonoaudiológico.

A coleta de dados foi realizada por meio da aplicação do protocolo de Avaliação Fonológica da Criança (AFC), de Yavas, Hernadorema e Lamprecht (2002). A avaliação foi criada com o objetivo de eliciar uma amostra representativa da fala por meio de nomeação espontânea. $\mathrm{O}$ instrumento é composto de cinco ilustrações temáticas: Veículos, Sala, Banheiro, Cozinha e Zoológico, que compõem os 125 itens da AFC.

A AFC foi aplicada no ambiente escolar, individualmente, em horário delimitado pela escola, com autorização prévia da Secretaria de Educação do Município e assinatura do Termo de Consentimento Livre e Esclarecido pelos pais ou responsáveis. Com base nos questionários devidamente respondidos pelos responsáveis, as crianças selecionadas foram submetidas à triagem de motricidade orofacial, com o objetivo de descartar da amostra as que apresentassem alterações nos órgãos fonoarticulatórios, descartando assim possíveis desvios fonéticos.

Os desenhos temáticos foram apresentados a cada criança individualmente e suas produções espontâneas foram gravadas e posteriormente transcritas na folha de registro que acompanha o protocolo de avaliação. Foi considerada a classificação dos principais processos fonológicos de acordo com a literatura e a fala foi avaliada quanto à ocorrência dos processos fonológicos alterados. Considerou-se desvio fonológico nas crianças que apresentaram menos de $86 \%$ de acertos, conforme descrito por Yavas, Hernanorema e Lamprecht (2001). Os processos fonológicos que foram avaliados são: redução de encontro consonantal, apagamento da sílaba final, apagamento da líquida final, apagamento da fricativa final, apagamento de líquida intervocálica, apagamento de líquida inicial, dessonorização de obstruintes, anteriorização, substituição de líquida, plosivização e posteriorização.

Esta pesquisa foi submetida à apreciação e aprovada pelo Comitê de Ética em Pesquisa da ULBRA sob protocolo (2010-234H). A coleta de dados só ocorreu após esta aprovação.

Os dados obtidos no estudo foram armazenados em um banco de dados no programa Excel. Foi realizada uma análise estatística descritiva e uma analise teórica estimando a prevalência de desvio fonológico na população total estudada em função de sexo e idade, bem como o cálculo da prevalência específica de cada processo fonológico alterado e sua relação com as variáveis em estudo. Os dados foram analisados por meio de tabelas estatísticas descritivas. Para comparação da presença de desvio fonológico e dos processos fonológicos entre os sexos e idade foi utilizado o teste Qui-quadrado. $\mathrm{Na}$ análise estatística foi utilizado o programa Statistical Package for Social Research (SPSS). Foram considerados estatisticamente significantes os achados com valor de $p \leq 0,05$.

\section{RESULTADOS}

Dentre as 60 crianças participantes da amostra, $27(45 \%)$ eram do sexo feminino e $33(55 \%)$ do sexo masculino. Deste total, $20(33,3 \%)$ tinham 4 anos de idade, $32(53,3 \%) 5$ anos e $8(13,3 \%)$ tinham 6 anos. A idade média dos participantes desta pesquisa foi de 4,8 anos.

A prevalência de desvio fonológico na amostra deste estudo foi de $55 \%$, totalizando 33 das 60 crianças estudadas. Quando comparado por sexo, observa-se uma maior prevalência para o gênero masculino, conforme Tabela 1.

Tabela 1 - Prevalência de desvio fonológico por sexo

\begin{tabular}{ccc}
\hline \multirow{2}{*}{ Sexo } & \multicolumn{2}{c}{ Desvio fonológico } \\
\cline { 2 - 3 } & Presença & Ausência \\
\hline Feminino & 48,1 & 51,9 \\
Masculino & 60,6 & 39,4 \\
\hline Total & 55,0 & 45,0 \\
\hline
\end{tabular}

Teste Qui-quadrado * "p" $\leq 0,05$. 
$\mathrm{Na}$ relação entre a idade e a prevalência de desvio fonológico observou-se maior prevalência aos cinco anos de idade $(59,4 \%)$, como pode-se observar na Tabela 2.

Tabela 2 - Prevalência de desvio fonológico por idade

\begin{tabular}{ccc}
\hline \multirow{2}{*}{ Idade } & \multicolumn{2}{c}{ Desvio fonológico } \\
\cline { 2 - 3 } & Presença & Ausência \\
\hline 4 & 50,0 & 50,0 \\
5 & 59,4 & 40,6 \\
6 & 50,0 & 50,0 \\
\hline Total & 55,0 & 45,0 \\
\hline
\end{tabular}

Teste Qui-quadrado * "p" $\leq 0,05$.

Os processos fonológicos mais usados pelas crianças podem ser observados na Tabela 3 . Nota-se que os quatro processos fonológicos mais utilizados foram: redução de encontro consonantal, com prevalência de $46,7 \%$ (28 crianças), apagamento da líquida final $40 \%$ (24 crianças), substituição de líquida $30 \%$ (18 crianças) e anteriorização $26,7 \%$ (16 crianças).

$\mathrm{Na}$ Tabela 4 pode-se observar os processos fonológicos comparados com a idade das crianças. Os mais frequentes aos quatro anos de idade foram: redução de encontro consonantal (45\%), apagamento de líquida final (35\%), anteriorização (30\%), apagamento de sílaba átona e substituição de líquida (25\%); aos cinco anos de idade observou-se: redução de encontro consonantal (50\%), apagamento de líquida final $(43,8 \%)$, substituição de líquida (37,5\%), e anteriorização $(28,1 \%)$; e aos seis anos: redução de encontro consonantal e apagamento da líquida (37,5\%).

$\mathrm{Na}$ relação entre os sexos e processos fonológicos constatou-se maior prevalência da redução de encontro consonantal para o sexo masculino, sendo $(40,7 \%)$ para o sexo feminino e $(51,5 \%)$ para o sexo masculino. $O$ apagamento da líquida final foi $(37 \%)$ no sexo feminino e $(42,4 \%)$ no sexo masculino, e a substituição de líquida foi $(33,3 \%)$ no sexo feminino e $(27,3 \%)$ no sexo masculino. Estes processos foram observados como os mais freqüentes. A prevalência do processo fonológico de apagamento de sílaba átona quanto ao sexo teve associação significante, onde se observa que o sexo masculino possui maior presença sendo $(21,2 \%)(p=0,050)$, conforme Tabela 5 .

\section{DISCUSSÃO}

No Brasil ainda são poucos os estudos sobre a prevalência dos desvios fonológicos, distúrbio causado por uma desordem linguística que se caracteriza pelo uso de padrões anormais de expressão da linguagem falada. O desvio fonológico é representado por crianças as quais apresentam

Tabela 3 - Ocorrência dos processos fonológicos nas crianças avaliadas

\begin{tabular}{lcccc}
\hline \multirow{2}{*}{ Variável } & \multicolumn{2}{c}{ Presença } & \multicolumn{2}{c}{ Ausência } \\
\cline { 2 - 5 } & No casos & \% & № casos & \% \\
\hline Processos Fonológicos & & & 32 & 53,3 \\
Redução de encontro consonantal & $\mathbf{2 8}$ & $\mathbf{4 6 , 7}$ & 52 & 86,7 \\
Apagamento de Silaba átona & $\mathbf{8}$ & $\mathbf{1 3 , 3}$ & 54 & 90,0 \\
Apagamento da Fricativa final & $\mathbf{6}$ & $\mathbf{1 0 , 0}$ & 36 & 60,0 \\
Apagamento da Líquida Final & $\mathbf{2 4}$ & $\mathbf{4 0 , 0}$ & 56 & 93,3 \\
Apagamento da Líquida Intervocálica & $\mathbf{4}$ & $\mathbf{6 , 7}$ & 56 & 93,3 \\
Apagamento da Líquida Inicial & $\mathbf{4}$ & $\mathbf{6 , 7}$ & 56 & 93,3 \\
Dessonorização & $\mathbf{4}$ & $\mathbf{6 , 7}$ & 44 & 73,3 \\
Anteriorização & $\mathbf{1 6}$ & $\mathbf{2 6 , 7}$ & $\mathbf{3}, 3$ \\
Substituição de Líquida & $\mathbf{1 8}$ & $\mathbf{3 0 , 0}$ & 42 & 70,0 \\
Semivocalização & $\mathbf{8}$ & $\mathbf{1 3 , 3}$ & 52 & 86,7 \\
Plosivização & $\mathbf{1}$ & $\mathbf{1 , 7}$ & 59 & 98,3 \\
Posteriorização & $\mathbf{4}$ & $\mathbf{6 , 7}$ & 56 & 93,3 \\
Cultural & $\mathbf{2}$ & $\mathbf{3 , 3}$ & 58 & 96,7 \\
\hline
\end{tabular}

Teste Qui-quadrado 
Tabela 4 - Ocorrência de processos fonológicos por idade nas crianças com desvio fonológico

\begin{tabular}{|c|c|c|c|c|c|}
\hline \multirow{2}{*}{ Processo Fonológico } & \multirow{2}{*}{ Resultado } & \multicolumn{3}{|c|}{ Idade } & \multirow{2}{*}{$\mathbf{p}$} \\
\hline & & 4 anos & 5 anos & 6 anos & \\
\hline \multirow[t]{2}{*}{ Redução de encontro consonantal } & Presença & 45,0 & 50,0 & 37,5 & 0,804 \\
\hline & Ausência & 55,0 & 50,0 & 62,5 & \\
\hline \multirow[t]{2}{*}{ Apagamento de Silaba átona } & Presença & 25,0 & 9,4 & & 0,134 \\
\hline & Ausência & 75,0 & 90,6 & 100,0 & \\
\hline \multirow[t]{2}{*}{ Apagamento da Fricativa final } & Presença & 15,0 & 9,4 & & 0,482 \\
\hline & Ausência & 85,0 & 90,6 & 100,0 & \\
\hline \multirow[t]{2}{*}{ Apagamento da Líquida Final } & Presença & 35,0 & 43,8 & 37,5 & 0,812 \\
\hline & Ausência & 65,0 & 56,3 & 62,5 & \\
\hline \multirow[t]{2}{*}{ Apagamento da Líquida Intervocálica } & Presença & 5,0 & 9,4 & & 0,595 \\
\hline & Ausência & 95,0 & 90,6 & 100,0 & \\
\hline \multirow[t]{2}{*}{ Apagamento da Líquida Inicial } & Presença & 10,0 & 6,3 & & 0,626 \\
\hline & Ausência & 90,0 & 93,8 & 100,0 & \\
\hline \multirow[t]{2}{*}{ Dessonorização } & Presença & 5,0 & 6,3 & 12,5 & 0,765 \\
\hline & Ausência & 95,0 & 93,8 & 87,5 & \\
\hline \multirow[t]{2}{*}{ Anteriorização } & Presença & 30,0 & 28,1 & 12,5 & 0,616 \\
\hline & Ausência & 70,0 & 71,9 & 87,5 & \\
\hline \multirow[t]{2}{*}{ Substituição de Líquida } & Presença & 25,0 & 37,5 & 12,5 & 0,323 \\
\hline & Ausência & 75,0 & 62,5 & 87,5 & \\
\hline \multirow[t]{2}{*}{ Semivocalização } & Presença & 15,0 & 15,6 & & 0,491 \\
\hline & Ausência & 85,0 & 84,4 & 100,0 & \\
\hline \multirow[t]{2}{*}{ Plosivização } & Presença & 5,0 & & & 0,362 \\
\hline & Ausência & 95,0 & 100,0 & 100,0 & \\
\hline \multirow[t]{2}{*}{ Posteriorização } & Presença & 5,0 & 6,3 & 12,5 & 0,765 \\
\hline & Ausência & 95,0 & 93,8 & 87,5 & \\
\hline \multirow[t]{2}{*}{ Cultural } & Presença & 5,0 & & 12,5 & 0,186 \\
\hline & Ausência & 95,0 & 100,0 & 87,5 & \\
\hline
\end{tabular}

Teste Qui-quadrado * "p” $\leq 0,05$.

alterações na fala na ausência de determinados fatores etiológicos ${ }^{4,6,11}$. A criança com desvio fonológico não possui um problema orgânico detectável, mas apresenta um sistema fonológico diferente da norma, podendo também apresentar um inventário fonético incompleto em relação ao padrão da sua comunidade linguística ${ }^{11,12}$.

A prevalência de alterações fonológicas encontradas nesse estudo foi de $55 \%$, semelhante a encontrada em um estudo realizado em pré-escolares de Belo Horizonte (MG), onde a amostra foi de crianças com idades semelhantes às desta pesquisa, que apresentou uma prevalência de $51,6 \%$ de alterações fonológicas. ${ }^{5}$ Porém, quando comparado a outros estudos realizados em Canoas -RS onde a faixa etária estudada foi de cinca a doze anos , Vale do Paraíba (RJ) na faixa de etária de sete anos,e Bahia na faixa etária de quatro a seis anos, onde a prevalência varia de $8,27 \%$ a
$24,6 \%$, observa-se a alta prevalência encontrada em nosso estudo ${ }^{3,4,9,12}$. Esta diferença pode ser explicada pela variabilidade de idade dos sujeitos avaliados nas pesquisas, pois de acordo com a literatura a aquisição do sistema fonológico ocorre de maneira gradual até o estabelecimento do mesmo, que pode estar praticamente completo aos cinco anos, mas também pode-se estender dos quatro aos sete anos de idade ${ }^{5,7-9}$. Os estudos que resultaram em uma menor prevalência apresentaram amostra com crianças com mais de seis anos ${ }^{3,4,9}$. A alta prevalência encontrada na presente pesquisa também pode ser explicada por um viés de seleção denominado "variável de influência"13, pois pode favorecer a autorização dos pais de crianças com alterações de fala, por estarem mais sensibilizados para aceitar a participação de seu filho no estudo, com o intuito de receber ajuda para o caso ${ }^{13,14}$. 
Indrusiak CS, Rockenbach SP

Tabela 5 - Ocorrência de processos fonológicos por sexo nas crianças com desvio fonológico

\begin{tabular}{|c|c|c|c|c|}
\hline \multirow{2}{*}{ Processos Fonológicos } & \multirow{2}{*}{ Resultado } & \multicolumn{2}{|c|}{ Sexo } & \multirow{2}{*}{$\mathbf{p}$} \\
\hline & & Feminino & Masculino & \\
\hline \multirow[t]{2}{*}{ Redução de encontro consonantal } & Presença & 40,7 & 51,5 & 0,446 \\
\hline & Ausência & 59,3 & 48,5 & \\
\hline \multirow[t]{2}{*}{ Apagamento de Silaba átona } & Presença & 3,7 & 21,2 & $0,050^{*}$ \\
\hline & Ausência & 96,3 & 78,8 & \\
\hline \multirow[t]{2}{*}{ Apagamento da Fricativa final } & Presença & 3,7 & 15,2 & 0,209 \\
\hline & Ausência & 96,3 & 84,8 & \\
\hline \multirow[t]{2}{*}{ Apagamento da Líquida Final } & Presença & 37,0 & 42,4 & 0,793 \\
\hline & Ausência & 63,0 & 57,6 & \\
\hline \multirow[t]{2}{*}{ Apagamento da Líquida Intervocálica } & Presença & 3,7 & 9,1 & 0,620 \\
\hline & Ausência & 96,3 & 90,9 & \\
\hline \multirow[t]{2}{*}{ Apagamento da Líquida Inicial } & Presença & 3,7 & 9,1 & 0,620 \\
\hline & Ausência & 96,3 & 90,9 & \\
\hline \multirow[t]{2}{*}{ Dessonorização } & Presença & 7,4 & 6,1 & 0,835 \\
\hline & Ausência & 92,6 & 93,9 & \\
\hline \multirow[t]{2}{*}{ Anteriorização } & Presença & 29,6 & 24,2 & 0,771 \\
\hline & Ausência & 70,4 & 75,8 & \\
\hline \multirow[t]{2}{*}{ Substituição de Líquida } & Presença & 33,3 & 27,3 & 0,778 \\
\hline & Ausência & 66,7 & 72,7 & \\
\hline \multirow[t]{2}{*}{ Semivocalização } & Presença & 18,5 & 9,1 & 0,448 \\
\hline & Ausência & 81,5 & 90,9 & \\
\hline \multirow[t]{2}{*}{ Plosivização } & Presença & & 3,0 & 0,550 \\
\hline & Ausência & 100,0 & 97,0 & \\
\hline \multirow[t]{2}{*}{ Posteriorização } & Presença & 7,4 & 6,1 & 0,614 \\
\hline & Ausência & 92,6 & 93,9 & \\
\hline \multirow[t]{2}{*}{ Cultural } & Presença & & 6,1 & 0,497 \\
\hline & Ausência & 100,0 & 93,9 & \\
\hline
\end{tabular}

Teste Qui-quadrado * "p" $\leq 0,05$.

Analisando o desvio fonológico por sexo, pode-se perceber que, embora não seja significante, ocorreu em maior número para os meninos que para as meninas. Outros estudos, realizados nos estados do Rio Grande Sul, Minas Gerais e Rio de Janeiro também apontam a maior prevalência de alterações de fala em indivíduos do sexo masculino ${ }^{1,3,4,9}$. A literatura aponta como justificativa para a maior prevalência de alterações de linguagem no sexo masculino, o fato de a aquisição e desenvolvimento da linguagem entre meninos e meninas ocorrer de forma diferente, ou o cérebro do indivíduo do sexo masculino apresentar uma maturação mais lenta. Pois existem diferenças sexuais na cronologia da mielinização neuronal, sendo ela mais precoce em meninas em áreas relacionadas a linguagem, o que pode explicar a superioridades no desenvolvimento das habilidades lingüísticas. Porém ainda não há nenhum estudo que comprove a relação entre alterações fonológicas e a predominância do sexo masculino 1,9, 14 .

É fato documentado que as crianças adquirem a maioria dos sons da sua língua até cinco ou seis anos de idade, mas algumas não conseguem aprender os padrões de sons até esta idade, apesar de suas aptidões cognitivas e motoras normais $5,7,10,16$. Embora não se tenha observado diferenças significantes entre as idades, quando foi analisada a prevalência de desvio fonológico nas idades de quatro a seis anos, quando se observou uma maior prevalência na faixa etária dos cinco anos. Este resultado corrobora pesquisa semelhante realizada em Salvador (BA), onde também foi verificada maior prevalência nesta faixa etária ${ }^{12}$. Outros estudos, embora com diferentes faixas etárias estudadas, também demonstram maior prevalência aos cinco anos $1,3,14$. Esses resultados permitem verificar que não há um padrão de aumento e diminuição 
do distúrbio conforme a idade. Apesar de a maior prevalência encontrada seja para a idade de cinco anos, o momento de estabelecimento do sistema fonológico é controverso, pois a literatura refere que o estabelecimento deste pode se estender dos quatro aos sete anos de idade $3,4,5,7-10,12,15$.

Um modelo de análise fonológica muito utilizado na descrição do sistema fonológico é o dos processos fonológicos. Referem-se a simplificações de regras fonológicas que envolvem uma sequência de sons da fala que ocorrem durante o desenvolvimento da linguagem oral ${ }^{5,6,10,17-20}$. Não se tem um consenso do número de processos fonológicos possíveis ${ }^{5,10,11,1}$

Foi observado, durante a realização da pesquisa, que crianças sem desvio fonológico apresentaram processos fonológicos alterados. Todavia para afirmar que uma criança já adquiriu um determinado segmento ou estrutura silábica não é necessário que $100 \%$ da produção esteja correta. Produções inadequadas podem apresentar resquícios de etapas fonológicas já superadas ou ainda simples lapsos de fala, não caracterizando necessariamente um (ou um quadro de) desvio fonológico.

Nesta pesquisa encontrou-se com maior prevalência os processos fonológicos de redução de encontro consonantal $(46,7 \%)$, apagamento da líquida final (40\%), anteriorização $(26,7 \%)$ e substituição de líquida (30\%), o que coincide com outros estudos realizados com crianças da mesma faixa etária e de faixas etárias próximas a pesquisada $4,5,12,17,18$. É importante ressaltar que não foram diferenciados nesse estudo o ponto articulatório do fonemas. Os processos fonológicos mais encontrados no estudo estão referidos na literatura como os últimos a serem adquiridos, o que confirma o achado de nossa pesquisa, demonstrando maior prevalência para estes processos 4,5,9,10,12,16, cuja ordem de ocorrência coincide com a sequência de aquisição da maioria das crianças com desenvolvimento fonológico normal. É importante ressaltar que não foram diferenciadas neste estudo as líquidas laterais das líquidas não laterais.

A literatura refere que a cronologia dos processos fonológicos ocorre entre um ano e seis meses e cinco anos de idade 5,9,16,18. Existem controvérsias no que diz respeito à idade de supressão dos processos fonológicos. Como pode ser observado na Tabela 4, a maior prevalência encontrada foi de redução de encontro consonantal na idade de cinco anos, que, de acordo com a literatura estudada, deveria estar superado na cronologia normal dos processos fonológicos ${ }^{9,15}$. Pode se observar que os processos fonológicos mais prevalentes foram os mesmos para todas as faixas etárias estudadas. Os resultados estão de acordo com os achados de outras pesquisas de mesma faixa etária ${ }^{12}$. Foi observado que aos quatro anos as crianças apresentaram mais de um processo fonológico alterado, sendo os mais frequentes: a redução de encontro consonantal, o apagamento de líquida final, a anteriorização e a substituição de líquida e apagamento de sílaba átona. Segundo a literatura, aos quatro anos estes processos, com exceção da redução de encontro consonantal, já deveriam estar superados 10,16. Os achados desta pesquisa corrobora a literatura, a qual relata os processos fonológicos envolvendo as líquidas laterais e não laterais. A literatura refere que os processos fonológicos com líquidas são os últimos a serem superados na cronologia do desenvolvimento da aquisição da linguagem oral ${ }^{7,8,12,18,20}$. Os resultados desta pesquisa mostram que o desenvolvimento fonológico das crianças estudadas se dá em ordem semelhante à ordem de aquisição fonológica de crianças sem desvio fonológico, entretanto, de forma mais tardia ${ }^{5,9,16}$.

Pode ser visto nos resultados deste estudo que a prevalência dos processos fonológicos de redução de encontro consonantal e apagamento da líquida final foi maior para o sexo masculino que para o feminino, o que concorda com outras pesquisas ${ }^{4,12}$, enquanto os processos fonológicos de anteriorização e substituição de líquida se mostraram com maior prevalência para o sexo feminino. Verificouse ainda uma prevalência estatisticamente significante para o apagamento de sílaba átona nos indivíduos do sexo masculino, o que não foi encontrado na bibliografia estudada.

\section{CONCLUSÃO}

Os resultados deste estudo mostraram que, na amostra estudada, a prevalência de desvio fonológico foi de $55 \%$, com maior ocorrência para o sexo masculino. Na faixa etária de cinco anos, observouse a maior prevalência de desvio fonológico.

Os processos fonológicos que se mostraram mais prevalentes na população estudada foram de redução de encontro consonantal, apagamento da líquida final, substituição de líquida e anteriorização. $O$ processo mais frequente por idade foi a redução de encontro consonantal aos 5 anos. Quando investigaram-se os processos fonológicos por sexo observou-se que o apagamento de sílaba átona foi significante para o sexo masculino.

Os índices de alterações e caracterização por idade desta pesquisa revelam uma alta prevalência de desvio fonológico, o que está de acordo com outros estudos, apontando a necessidade de inserção de programas públicos de promoção e prevenção à saúde da comunicação humana, junto às escolas de educação infantil e unidades de saúde. 


\begin{abstract}
Purpose: to check the prevalence of phonological deviations and processes according to gender and age. Method: statistic and descriptive analysis of phonological evaluation of Yavas, Hernandorema and Lamprecht (2002). This was carried through in 60 kindergarden schools from 4 to 6 year old in municipal schools in Canoas - RS. The sample consisted of children whose parents did not report any auditory and neurological alterations and syndromes in the questionnaire. They did not specify if these children had already taken phonological treatment or phonetic deviations. Results: the prevalence of phonological deviations was 55\% and most of them were males. When they were 5 year old, we found major prevalence of phonological deviations. The most found phonological processes were a consonantal join of reduction $(46,7 \%)$, final liquid erase $(40 \%)$ and liquid substitution $(30 \%)$. The relations of the phonological prevalence process with gender were demonstrated similar in certain ways, except from the atonic syllabus for the male gender. Conclusion: the high prevalence of phonological deviations shows the necessity of public programs and health prevention for human communication.
\end{abstract}

KEYWORDS: Prevalence; Speech, Language and Hearing Sciences; Language

\section{REFERÊNCIAS}

1. César AM, Maksud SS. Caracterização da demanda de fonoaudiologia do serviço público municipal de Ribeirão das Neves- MG. Rev. CEFAC. Jan- Mar, 2007; 9 (1): 133-8.

2. Santos GG, Melo PDF, Diniz JM, Teixeira GBP. A importância do diagnóstico diferencial das alterações de fala: enfoque fonoaudiológico. J Bras Fonoaudiol. 2003; 4 (16): 186-92.

3. Goulart BNG, Chiari, BM. Prevalência de desordens de fala em escolares e fatores associados. Rev Saúde Pública. 2007; 41 (5): 726-31.

4. Patah LK, Takiuchi N. Prevalência das alterações fonológicas uso dos processos fonológicos em escolares aos 7 anos. Rev CEFAC. Abr- Jun, 2008; 10 (2): 158- 67.

5. Vitor RM, Cardoso-Martins C. O desenvolvimento fonológico de crianças pré- escolares da Região Noroeste de Belo Horizonte. Psicologia em Rev. Jun, 2007; 13 (2): 383- 98.

6. Spíndola RA, Payão LMC, Bandini HHM. Abordagem fonoaudiológica em desvios fonológicos fundamentada na hierarquia dos traços distintivos e na consciência fonológica. Rev CEFAC. Abr-jun, 2007; 9 (2): 180-9.

7. Mota HB, Kaminski TI, Nepomuceno MRF, Athayde ML. Alterações no vocabulário expressivo de crianças com desvio fonológico. Rev Soc Bras Fonoaudiol. 2009; 14 (1): 41-7.

8. Ferrante C, Borsel JV, Pereira MMB. Aquisição fonológica em crianças de classe econômica alta. Rev CEFAC. 2008; 10 (4): 452-60.
9. Rockenbach SP. Prevalência de distúrbios de fala em crianças da primeira série de Escolas Municipais de Esteio. [tese] Disponível em: http:// hdl.handle.net/10183/779. Acesso em: 24 mar. 2010 às $15 \mathrm{~h}$.

10. Ferrante C, Borsel JV, Pereira MMB. Análise dos processos fonológicos em crianças com desenvolvimento fonológico normal. Rev Soc Bras Fonoaudiol. 2009; 14 (1): 36-40.

11. Yavas M, Hernandorema CLM, Lamprecht RR. Avaliação fonológica da criança: reeducação e terapia. $2^{a}$ Ed. Porto Alegre: Ed. Artmed; 2002.

12. Cavalheiro BG. Prevalência de desvio fonológico em crianças de 4 a 6 anos de Escolas Municipais de Salvador- BA. Rev Soc Bras Fonoaudiol. 2008; 13 (4): 415.

13. Andrade CRF. Prevalência das desordens idiopáticas da fala e da linguagem em crianças de um a onze anos de idade. Rev de Saúde Pública. Out, 1997; 31 (5):495-501.

14. Barros PML, Oliveira PN. Perfil dos pacientes atendidos no setor de fonoaudiologia de um serviço público de Recife-PE. Rev CEFAC. Jan-fev, 2010; 12 (1):128- 33.

15. Miranda MC, Muszkat M. Neuropsicologia do desenvolvimento. In: Andrade VM, Santos FH, Bueno OFA. Neuropsicologia hoje. São Paulo: Artes Médicas, 2004. p.211-24.

16. Yavas MS. Desvio Fonológico em Crianças: teoria, pesquisa e tratamento. $1^{\text {a }}$ ed. Porto Alegre: Ed Mercado Aberto;1990. 
17. Wertzner HF, Pagan LO, Galea DES, Papp ACCS. Características fonológicas de crianças com transtorno fonológico com e sem histórico de otite média. Rev Soc Bras Fonoaudiol. 2007; 12 (1): 41-7.

18. Nunes DA, Payão LMC, Costa PCC. Desvio fonológico na Educação Infantil. Rev CEFAC. MarAbr 2010;12 (2): 331-6.
19. Pagan LO, Wertzner HF. Análise acústica das consoantes líquidas do Português Brasileiro em crianças com transtorno fonológico. Rev Soc Bras Fonoaudiol. 2007; 12 (2): 106-13.

20. Wertzner HF, et al. Ocorrência de otite média e infecções de vias aéreas superiores em crianças com distúrbio fonológico. Rev Soc Bras Fonoaudiologia. 2002; 7 (1):32-9.
http://dx.doi.org/10.1590/S1516-18462012005000011

RECEBIDO EM: 01/3/2011

ACEITO EM: 18/08/2011

Endereço para correspondência:

Camila dos Santos Indrusiak

Rua Angelo Possebom no 36/ 702, Canoas - RS

CEP: 92310-140

E-mail: cacaindrusiak@ hotmail.com 